\title{
ST-Segment Elevation Myocardial Infarction in Dialysis and Renal Transplant Patients. How Does It Compare to That in the General Population?
}

\author{
Islam A. Bolad \\ Krannert Institute of Cardiology, Indiana University School of Medicine, Indianapolis, Ind., USA
}

Cardiovascular death is the leading cause of mortality in patients with end-stage renal disease (ESRD). It is also the leading cause of death in renal transplant patients [1]. Over the past few decades, multiple studies showed that the incidence of acute myocardial infarction (AMI) is high in patients with ESRD and that the incidence of AMI is directly related to the reduction in kidney function, with increased mortality in this patient population [2]. It has also been shown that the utilization of urgent coronary revascularization in the form of either primary percutaneous coronary intervention or coronary artery bypass graft surgery, to preserve myocardial tissue, is unfortunately low in this high risk patient population compared to the general population [2]. AMI is a broad term which includes 5 different types as outlined in the Universal Definition of Myocardial Infarction expert writing group consensus document [3]. Electrocardiographically, AMI shows on the tracing as either ST-segment elevation (STEMI) or ST-segment depression or no ST-segment change. Acute and complete blockage of a coronary artery or its branch usually reflects on the electrocardiogram as STEMI. There has been paucity of studies looking into STEMI in patients with ESRD on dialysis and in renal transplant patients [4].

In this issue, Sakhuja et al. [5] used the National Inpatient Sample Database and investigated the incidence of STEMI in patients with ESRD on maintenance dialysis and in renal transplant patients during a 5-year period, and

\section{KARGER}

E-Mail karger@karger.com

www.karger.com/ajn compared it to other patients with STEMI which they categorized as the general population. There were 882,447 STEMI hospitalizations, 11,383 were in ESRD patients on maintenance dialysis and 1,076 were in renal transplant patients. They found that incidences of STEMI in dialysis patients and renal transplant recipients were 7.7 and 1.3 times, respectively, higher than in the general population. The incidence declined over the study period, although it did not reach statistical significance in the renal transplant patients; the decline was greatest in the maintenance dialysis patients compared to the general population. The utilization of revascularization procedures was lowest in patients on maintenance dialysis compared to the other 2 categories. The in-hospital mortality was highest in patients on dialysis; being on dialysis and having renal transplant were independent predictors of in-hospital mortality.

Data on the incidence, management and outcomes of STEMI in patients with ESRD on dialysis and renal transplant patients are limited. The incidence and severity of coronary artery disease increase as the glomerular filtration rates decline. Coronary disease in patients with impaired kidney function and ESRD usually has a pattern of multi-vessel involvement with coronary calcification. Although the incidence of AMI is known to be elevated in patients with ESRD, Sakhuja et al. [5] has now demonstrated that the incidence of STEMI per se is also elevated in patients with ESRD on dialysis. This is not surprising since patients with ESRD have high incidence of comor- 
bid conditions such as diabetes mellitus and hypertension, in addition to less well established conditions such as low concentrations of high density lipoprotein cholesterol, increased calcium phosphate product, hyperparathyroidism, decreased nitric oxide activity and hyperhomocysteinemia. What is also interesting is that the authors demonstrated that although kidney transplantation reduced the incidence of STEMI, it did not reduce it to a level to be in line with the incidence seen in the general population. This is possibly related to pre-transplant comorbid conditions in this patient population, the effect of immunosuppressive medications and the exposure after transplantation to a variety of traditional and nontraditional risk factors.

The incidence of STEMI in the general population in the United States has been declining over the years [6]. In this multi-institutional population-based observational study, Sakhuja et al. [5] showed that the incidence of STEMI in ESRD on maintenance dialysis also declined during their study period. This might be related to better risk factors modification and more aggressive use of cardioprotective medications. There was also a trend toward reduced incidence of STEMI in renal transplant patients, although this did not reach statistical significance. Dialysis patients presenting with AMI in general have low utilization of revascularization procedures compared to the general population [7]. This is possibly due to delay in the diagnosis and presentation, atypical presentation symptoms and higher comorbid conditions. The data from the current study show that this underutilization of revascularization extends to STEMI patients on dialysis, with only slightly less revascularization underutilization in kidney transplant patients. The in-hospital mortality was highest in patients on dialysis followed by patients with renal transplant; the long-term outcomes of these patients are unknown.
The study has few limitations. ICD-9-CM codes were used for patient selection, and although these have high sensitivity and specificity, they are by no means $100 \%$ inclusive and depend largely on the expertise of the coders. Being a retrospective observational study, there is the likelihood of selection bias and certain level of details like symptom onset to balloon times, do not resuscitate status and readmissions are not available. The database used does not include patients from the Veterans Affairs Hospitals. Outcomes from the National Inpatient Sample database are limited to in-patient events; the causes of death are undifferentiated and patients who did not make it to the hospital are not included.

This large observational study [5] provides us with important information about the STEMI incidence, utilization of revascularization and in-hospital mortality in ESRD on dialysis patients and in renal transplant recipients as compared to STEMI patients in the general population. It also raises questions that need to be answered in future studies. The reasons for the reduced utilization of revascularization procedures in this patient population are unfortunate, and factors leading to this need to be explored. It is not clear why the incidence of STEMI in renal transplant recipients is not declining over time as much as in patients on dialysis and in the general population. Long-term outcomes data of emergent revascularization of STEMI either by primary percutaneous coronary intervention or by coronary artery bypass graft surgery in this patient population are limited, and these outcomes need to be studied.

\section{Disclosure Statement}

The author has no conflict of interest to declare.

\section{References}

1 Briggs JD: Causes of death after renal transplantation. Nephrol Dial Transplant 2001;16: 1545-1549.

2 Fox CS, Muntner P, Chen AY, Alexander KP, Roe MT, Cannon CP, Saucedo JF, Kontos MC, Wiviott SD: Use of evidence-based therapies in short-term outcomes of ST-segment elevation myocardial infarction and non-STsegment elevation myocardial infarction in patients with chronic kidney disease: a report from the national cardiovascular data acute coronary treatment and intervention outcomes network registry. Circulation 2010; 121:357-365
3 Thygesen K, Alpert JS, Jaffe AS, Simoons ML Chaitman BR, White HD, et al: Third universal definition of myocardial infarction. J Am Coll Cardiol 2012;60:1581-1598.

4 Gupta T, Kolte D, Khera S, Harikrishnan P, Mujib M, Aronow W, Jain D, Cooper H, Frishman W, Bhatt D, Fonarow G, Panza J: Management and outcomes of ST-elevation myocardial infarction in renal transplant recipients versus end-stage renal disease patients on dialysis. J Am Coll Cardiol 2016;67: 650 .
5 Sakhuja A, Wright S, Schold J, McCarthy J, Williams A, Amer H, Albright R: National impact of maintenance dialysis or renal transplantation on outcomes following ST elevation myocardial infarction. Am J Nephrol 2016;44:329-338

6 Yeh RW, Sidney S, Chandra M, Sorel M, Selby JV, Go AS: Population trends in the incidence and outcomes of acute myocardial infarction. N Engl J Med 2010;362:2155-2165.

7 Charytan D, Mauri L, Agarwal A, Servoss S, Scirica B, Kuntz RE: The use of invasive cardiac procedures after acute myocardial infarction in long-term dialysis patients. Am Heart J 2006; 152:558-564. 